\title{
Linx
}

Revue des linguistes de l'université Paris X Nanterre

$7 \mid 1995$

Saussure aujourd'hui

\section{La coupure saussurienne}

\section{Claudine Normand}

\section{(2) OpenEdition \\ Journals}

Édition électronique

URL : http://journals.openedition.org/linx/1157

DOI : 10.4000/linx. 1157

ISSN : 2118-9692

\section{Éditeur}

Presses universitaires de Paris Nanterre

\section{Édition imprimée}

Date de publication : 1 septembre 1995

Pagination : 219-231

ISSN : 0246-8743

\section{Référence électronique}

Claudine Normand, «La coupure saussurienne », Linx [En ligne], 7 | 1995, mis en ligne le 13 juillet 2012, consulté le 21 avril 2019. URL : http://journals.openedition.org/linx/1157 ; DOI : 10.4000/linx.1157

Ce document a été généré automatiquement le 21 avril 2019

Département de Sciences du langage, Université Paris Ouest 


\title{
La coupure saussurienne
}

\author{
Claudine Normand
}

1 Que supposait cette métaphore quand elle ouvrait une recherche ? Quel rapport à l'objet se donnait ainsi dans l'éclat d'un geste tranchant? C'est ce que je voudrais éclairer.

2 Le rapport qu'avaient au Cours de linguistique générale ceux - dont j'étais - qui adoptèrent ce point de départ, dans les années 70, présente des traits particuliers qui le distinguent des rapports savants propres généralement aux études saussuriennes. En ce qui me concerne une de ses particularités est de s'être voulu non-savant ; c'est par ce trait que, dans l'histoire de la réception de Saussure, il a pu jouer quelque rôle.

3 Aujourd'hui que toute une production historique, biographique et/ou critique, se déploie au sujet des années 60-70, je tenterai à mon tour, à l'occasion de ce colloque, d'apporter quelques données aux historiens de ce temps. Ce rappel, lié à des moments et des lieux plus ou moins effacés aujourd'hui, sera évidemment très différent, dans ses dimensions comme dans sa démarche, des synthèses de Thomas Pavel (1988) ou de François Dosse (1991) ; ni le séduisant balayage philosophique du premier ni la mine d'informations sociohistoriques de l'autre ne relèvent de mon projet. Ce ne sera pas davantage un règlement de comptes, lourd de reniement tel qu'on peut en lire ça et là, commis par quelque acteur de ce théâtre qui veut aujourd'hui en écrire l'histoire en spectateur ironique et désabusé $;^{1}$ je ne prétends pas pour autant à l'objectivité de l'histoire : on ne peut, sur une période qu'on a vécue, qu'apporter un témoignage, rappeler certains débats et soumettre les souvenirs douteux qu'on en garde à la confrontation avec d'autres souvenirs et, surtout, avec les archives, les textes écrits à ce moment là.

\section{Saussure au programme}

Par « non-savant » je désigne un rapport longtemps naïf et direct au texte de Saussure tel que nous en disposions en cette fin des années 60 où je me suis trouvée, presque du jour au lendemain, sommée de le présenter à des étudiants de première année. Je voudrais attirer l'attention sur ce qui n'intéresse pas généralement les auteurs de panoramas et de synthèses : le moyen de diffusion massif et, par là, forcément porteur d'effets historiques, 
que représente le discours universitaire anonyme; dans le cas présent celui qui était tenu dans les universités de Lettres brusquement multipliées et chargées d'intégrer la linguistique « moderne » à leurs programmes, dès la première année. Je ne parle pas des professeurs célèbres, mais de ce discours obscur, voué à la mésinterprétation et à l'oubli, tenu dans les nombreuses salles où l'on ne distinguait plus, jusqu'à une date relativement récente, cours magistral et travaux dirigés.

Les textes que j'ai écrits dans ces années là, disons de 1970 à 1980, sont autant de jalons dans mon parcours d'enseignante, chacun ayant été suscité par la nécessité de reprendre tel ou tel thème saussurien qui, soudain, résistait à être clairement exposé: la valeur linguistique, l'arbitraire, le concept de langue...

6 On peut n'accorder à ces textes qu'un intérêt historique ; il reste que ce type d'archives, articles en quelque sorte de "circonstances ", concerne bien la réception de Saussure si l'on accepte de faire entrer dans cette histoire les répercussions multiples et très diverses de la lecture du CLG dans la linguistique du XXème siècle. Nombre d'étudiants des années 60 sont devenus linguistes et tous ont dû se déterminer par rapport à Saussure, dans la fidélité, le dépassement ou la rébellion. On en trouve encore aujourd'hui des effets dans les prises de position explicites "contre Saussure" chez certains pragmaticiens et sociolinguistes, comme s'il était encore nécessaire de répéter qu'« on en est enfin sorti », après avoir tant de fois affirmé que c'était fait et bien fait. "

7 Ainsi nous commentions Saussure : parfois lecture de lectures, celles de Benveniste, de Mounin, de Barthes... plus souvent lecture délibérément directe, négligeant les déjà trop nombreux commentaires pour interroger le texte lui-même. Mais quel texte? Evidemment la dite "vulgate », les notes de R. Godel, trop sophistiquées pour une lecture d'initiation, ne servant que de compléments occasionnels. C'est sous cette forme - que les jeunes chercheurs actuels se croient tenus de vilipender - que la pensée de Saussure a joué son véritable rôle historique, non seulement sur l'essentiel du mouvement dit structuraliste, avant et même après 1957 (Godel), mais aussi sur cette masse étudiante promise à des destins divers. Ainsi lorsque Sungdo Kim assure dans sa très savante thèse que « le CLG que l'on a lu et discuté est un CLG fictif » (36), je trouve de l'excès dans cette affirmation, car il s'agit d'un objet historique bien réel et qui a produit des effets comme tel; de plus, face à ce qu'il est convenu d'appeler la « vulgate » (ou, variante, « une sorte d'écriture sainte" selon R. Amacker cité par Kim ibid.), je vois pointer une forme de dévotion jalouse à l'authenticité, soutenue par la conviction un peu étrange qu'un sens aurait été délibérément déformé et la vérité emprisonnée, ainsi que le suggère d'ailleurs De Mauro :

Face aux problèmes de la formation du texte et, plus encore, de la formation de la pensée saussurienne elle-même, l'architecture unitaire imposée par les éditeurs s'effrite et croule; il en jaillit, problématique, authentique, vitale, la pensée de Saussure, libérée de ce que les éditeurs, avec les meilleures intentions du monde, lui avaient donné de dogmatique et de gratuit » (De Mauro, 408). Note curieusement romantique, sinon romanesque, dans ce scrupuleux travail d'édition !

9 Je ne plaide pas ici pour l'ignorance mais pour la reconnaissance d'objets distincts : d'une part le travail philologique, représenté avant tout par l'édition de R. Engler et sur quoi prend appui un travail interprétatif multiple se donnant pour horizon la véritable pensée de Saussure; d'autre part l'objet historique CLG, le seul connu de 1916 à 1957, qui a suscité nombre de comptes rendus critiques, a servi de référence à Hjelmslev comme à Benveniste, puis au mouvement de pensée beaucoup plus large que l'on connaît; seul 
texte, en outre, directement accessible au non spécialiste. Un troisième objet est concevable: Saussure pour la pensée d'aujourd'hui ${ }^{2}$; ce texte peut-il encore éclairer les grammairiens actuels et avoir des effets stimulants sur les philosophes et les psychanalystes? Je ne me prononcerai pas sur la grammaire mais pour ce qui est des autres spécialistes il est permis de penser qu'une lecture attentive de Saussure peut être encore fructueuse pour leur approche spécifique du langage ; le Saussure " authentique " est sans doute alors plus stimulant bien que réclamant plus d'effort de lecture.

\section{La vulgate de la vulgate}

Ce texte, donc, nous avions à lui faire un sort dans nos cours d'introduction à la linguistique. Un certain nombre d'idées couramment admises constituaient alors une autre espèce de vulgate, celle du commentaire; de cette lecture qui s'imposait ressortait que la nouveauté, jugée radicale, tenait à quelques dichotomies qui permettaient d'opposer l'ancien au nouveau (diachronie / synchronie) et le général, objet d'une science enfin possible, aux particularités relevant d'une approche subjective (langue / parole) ; s'y ajoutait l'énoncé méthodologique des deux axes nécessaires à la description du système ( syntagmes / associations) et l'énumération, parfaitement dogmatique, de principes très généraux: la langue est sociale, le signe unissant un signifiant et un signifié, est arbitraire, le signifiant est linéaire. On concluait éventuellement sur : « la langue est une forme et non une substance », sans trop savoir que faire de cette affirmation. Il était rare qu'on développe vraiment le terme système en système de rapports, de valeurs, de différences. L'essentiel se ramenait ainsi à ce qu'on appelait "concepts fondamentaux». Sur les relations de ces concepts, entre eux et avec les méthodes de description structurale, on n'était généralement pas très précis ; dans les cours, le plus souvent, on réglait d'abord la question des concepts saussuriens, éventuellement on les réservait au cours magistral quand il existait encore, puis on passait aux descriptions avec l'aide de Martinet, Bloomfield, Gleason... Cette schizophrénie n'a pas totalement disparu des cours de première année.

11 Ce résumé n'est pas une caricature ; qu'on se rappelle cette remarque de Ducrot (1968) :

«'Enfin Saussure vint!'

Trop de traités de linguistique publiés en Europe, ces trente dernières années, s'ouvrent par des déclarations à peine plus pudiques de forme et de contenu à peu près équivalent, qui ont largement contribué à rendre difficiles les relations entre les linguistes structuralistes d'une part, les philologues et les grammairiens de l'autre » (35).

12 Cette surestimation allait de pair avec une sous-estimation, dénoncée en 1972 par Mounin qui en voit la source chez Meillet, et avec la simplification que tout le " néo-saussurisme " (selon l'expression d'Amacker) dénonce depuis que les sources sont disponibles avec l'édition d'Engler. Le rôle de Meillet et de son collaborateur très proche, Vendryes, a été essentiel dans la mise en place immédiate de cette vulgate interprétative, grille de lecture qui a joué (et joue encore souvent) comme une évidence: l'essentiel, dans cette interprétation, est la distinction de la langue ("chose sociale») et de la parole individuelle; il faut cependant se garder de «l'abstraction» (en particulier dans l'opposition synchronie/ diachronie. cf. Meillet,1916). A plusieurs reprises Meillet insiste sur la continuité de ce qu'il appelle "l'Ecole de Paris», cette suite qui va de Bréal puis Saussure à lui-même et ses élèves ${ }^{3}$; et Vendryes systématise encore ce point de vue 
sociologique sur le CLG dans son compte-rendu de 1921, «Le caractère social du langage et la doctrine de F. Saussure », qui se conclut ainsi :

«Toutes les avenues de sa doctrine ramènent en effet vers la considération du langage comme un fait social (...). De la lecture de son livre on emporte la forte impression que la linguistique est essentiellement une science sociologique ».

13 Cette interprétation est reprise par Malmberg (1968) qui situe dans le CLG « l'introduction d'un point de vue sociologique» et en fait «l'apport vraiment original de sa doctrine »(69); elle perdure en 1980 chez G. Sampson qui intitule le chap. 2 de son ouvrage : "Saussure : language as social fact ». Il serait intéressant de réfléchir sur les raisons institutionnelles et personnelles qui ont pu installer en France une tradition interprétative ainsi simplifiée; toute la nouveauté du point de vue dit par Saussure "sémiologique " s'est trouvée traduite dans les termes sociologiques mis en place par Whitney dès les années 1870, repris par Meillet, aménagés selon son propre projet et largement diffusés, dès le début du XXème siècle (cf. en particulier son texte de 1906).

14 Cette interprétation était adoptée sans hésitation par la plupart des commentaires professoraux des années 60-70, même et surtout quand ils ignoraient que les propres thèses sociolinguistiques de Meillet étaient nettement antérieures à la parution du CLG.

\section{Lecture de lectures}

Ce n'est pas ce que je vais développer ici; je voulais seulement rappeler quelques conditions de notre rencontre avec le texte saussurien et que devant cette nouveauté, aussi catégoriquement située qu'elle était peu argumentée, il pouvait arriver qu'on s'interrogeât. Si nouveauté radicale il y avait - et j'en ai été dès la première lecture persuadée - pouvait-elle se résumer au caractère social de de la langue et / ou à l'arbitraire du signe? Ce deuxième point était donné comme un élément essentiel de l'originalité saussurienne. Une double question, me semblait-il, s'imposait: est-ce là le plus important? est-ce véritablement nouveau?

Pour la nouveauté, elle ne pouvait être vraiment évaluée que par rapport aux discours précédents et contemporains : que disaient-ils du « social » ou de l'« arbitraire »? Quant à l'intérêt principal de ce texte de 1916 que j'essayais de lire avec mes étudiants, sous les hésitations et confusions visibles malgré l'armature dogmatique "imposée», il m'apparaissait qu'il était ailleurs: dans le choix théorique d'une construction abstraite, aboutissant au concept de langue, définie comme système de valeurs, c'est à dire de différences.

17 C'était bien là ce qui devait être le plus difficile à faire entendre aux linguistes fascinés par les faits et persuadés qu'eux-mêmes ne leur surimposaient aucune hypothèse. La confirmation de cette intuition je la trouvais, a contrario, dans le commentaire de Meillet, mettant en garde contre l'abstraction et oubliant de mentionner la valeur. D'où l'idée de lire les autres comptes-rendus et commentaires pour mieux cerner ce qui pouvait alors surprendre ou paraître inacceptable ainsi que les oublis révélateurs d'incompréhension ${ }^{4}$.

\section{La théorie à l'ordre du jour}

Mais de quels instruments conceptuels disposions-nous alors pour analyser ces textes historiques et évaluer leur nouveauté ? Préciser ce point exige de revenir un peu plus 
longuement sur les conditions qui étaient alors celles de notre réflexion. J'ai déjà évoqué ce qu'était notre naïveté théorique lorsque, jeunes enseignants agrégés de Lettres classiques ou de Grammaire, nous décidâmes dans les années 60, de lire Saussure, comme nous y invitaient Lévi-Strauss et Barthes, alors même que déjà Ruwet nous avertissait que tout cela était dépassé par Chomsky5.

Dans ce cadre intervenaient les sollicitations de ce qu'on ne peut réduire ni à un engouement de mode parisienne ni à une imposture intellectuelle, toute l'effervescence théorique de ces années là, où ce qui se donnait comme réflexion et production « d'avantgarde » affirmait s'inscrire, par là même, dans un projet politique de changement radical. On n'explique rien, évidemment, en dénonçant aujourd'hui, comme le fait Courtine parmi d'autres, le caractère "illisible d'un certain style théorique", "la dramatisation épistémologique » et la prétention à « une position héroïque dans une lutte «théoricopolitique », dont Althusser s'est trouvé l'emblème (op.cit.). Cette lourde ironie dit surtout le reniement et le ressentiment personnel. Je le suivrai cependant sur un point, lorsque, parlant du «rapport de la linguistique comme science avec le projet politique de la gauche en France » dans cette période, il assure que la linguistique "est sortie du marxisme...en silence » et avec une certaine gêne, et qu'il serait intéressant de faire l'histoire de leurs rapports. La schématisation et les amalgames qu'il pratique montrent assez les limites d'un analyste trop impliqué dans la période analysée. A ce ressassement on préférera la lecture des archives de l'époque ${ }^{6}$.

Un exemple parmi tant d'autres dans le front des revues : les deux numéros de la Nouvelle Critique consacrés aux colloques de Cluny $(1968,1970)$; cette revue, sabordée en 1980 , était le mensuel officiel du parti communiste français. Un coup d'oeil sur les sommaires suffit pour voir que d'éminents représentants de la linguistique et de ce qui commençait à se dire sémiotique, étaient à Cluny où ils semblaient partager une sorte d'enthousiasme avec ce qui se donnait alors comme l'Avant-garde: Sollers, Kristeva, Meschonnic, Chevalier, Arrivé, bien d'autres.

21 Une histoire des relations des linguistes et de la gauche francaise (qui dans ces années-là tendait laborieusement vers son union, réalisée en 1972 et perdue dès 1978), concernerait aussi l'histoire propre de l'interprétation de Saussure. Son nom, en effet, était sans cesse brandi dans les débats théoriques qui nous agitaient. Selon les cas il se trouvait associé ou opposé à celui de Marx ; on le voyait pionnier d'une révolution scientifique ou pilier d'une idéologie réactionnaire. Il serait intéressant de consulter, si elles existent, les archives de la section linguistique du CERM (Centre d'études et de recherches marxistes) qui était alors le théâtre de débats souvent violents autour du nom de Saussure : vilipendé comme formaliste, positiviste, «objectiviste abstrait » par ceux qui se réclamaient, dans cette critique, de Volochinov (Bakhtine) et lui opposaient un programme de «linguistique sociale » repris à Meillet, il était défendu, au nom même du formalisme et de l'abstraction par ceux qui cherchaient à dégager sa nouveauté scientifique. En ce lieu, l'enjeu, on le devine, n'était pas seulement linguistique; la question des « deux sciences » n'était pas si ancienne; plus explicitement, encore qu'avec prudence, la position théorique sur la linguistique renvoyait à une position théorique plus globale et, par voie de conséquence, à "la ligne politique», véritable objet des discussions parmi ceux qu'on appelait les intellectuels du parti? 


\section{La coupure} la coupure. Ce terme, avec toute sa charge métaphorique, résumait le choix théorique de la discontinuité en Histoire, opposée aux notions classiques de développement graduel et de "précurseurs ». Ce qui chez Bachelard et Canguilhem jouait le rôle d'une hypothèse épistémologique appliquée à la physique et à la biologie, était soudain devenue, suite à sa rencontre avec la théorie politique chez Marx relu par Althusser, une sorte de mot d'ordre, le chiffre de la modernité. Cette grille de lecture par laquelle Althusser démarquait les écrits philosophiques du « jeune Marx » de ceux de sa maturité théorique ("scientifique»), devenait par exemple,dans le groupe constitué par le comité de rédaction de la revue Tel quel, un "programme " systématique de recherche et de production de textes théoriques. Sollers le formulait ainsi en 1967 ( $\left.{ }^{\circ} 31\right)$ :

«Une théorie d'ensemble, pensée à partir de l'écriture, demande à être élaborée »(3).

Quelques formules encore, qui nous frappaient:

«La théorie envisagée a sa source dans les textes de la rupture et de ceux qui sont susceptibles de l'annoncer et de la poursuivre" (le critère devant être «le coefficient de contestation théorique »).

Le « programme » se refermait sur l'affirmation suivante :

"Comme "conscience historique » elle (la théorie) se trouve nécessairement du côté de l'action révolutionnaire en cours »(7).

Sous leur solennité dogmatique et l'accent souvent qualifié de «terroriste » on retrouve, le développement de ce qu'annonçait Barthes, sur un autre ton, dès 1953 :

« Il y a dans toute écriture présente une double postulation : il y a le mouvement d'une rupture et celui d'un avènement, il $\mathrm{y}$ a le dessin même de toute situation révolutionnaire, dont l'ambiguïté fondamentale est qu'il faut bien que la Révolution puise dans ce qu'elle veut détruire l'image même de ce qu'elle veut posséder » (125).

Ce que Barthes appelait alors « l'utopie du langage » est devenu en 1967 un ordre du jour conquérant, au nom de la rupture.

Mais Saussure ne figurait pas aux côtés de Marx, Nietzsche et Freud dans les textes dits « de la rupture » que retenait Tel quel ; trop « modéré » sans doute, selon le terme de Pavel (op. cit p. 37) pour être adopté comme «subversif »; on se rappelle d'ailleurs que Derrida en 1967 l'avait marqué de soupçons durables. Il ne figure pas davantage dans le numéro d' Action poétique, autre revue en polémique avec Tel quel, où, quelques années plus tard E. Roudinesco fait le point sur ce qu'elle appelle "l'autre de la théorie", à savoir "l'idéologie qui sous-tend la plupart des doctrines poétiques et littéraires »; son article commence par une citation d'Althusser, suivie, entre autres, de Derrida, Foucault, Lacan et se termine ainsi :

«Ce numéro refuse à la fois l'esprit de tendance et le style du dogme, mais conforme aux meilleures couleurs de l'Agit-prop, il n'exclut ni le combat ni la prise de parti » (n53, 1973, p. 6).

Saussure en revanche intéressait beaucoup la revue Esprit, mais on n'y parlait pas à ce sujet de "coupure", seulement de "renversement des rapports entre système et histoire " (Ricoeur, 1963, n 11, 598); dès 1967 d'ailleurs, dans le numéro 5 de la même revue, c'est surtout à Benveniste que s'intéresse Ricoeur; il en attend le moyen du «retour à une intelligence intégrale du langage (...) penser l'unité de cela même que 
Saussure a disjoint, l'unité de la langue et de la parole.» (807); il ajoute que «la phénoménologie du sujet parlant trouve ici un appui solide » (810). Tout autre discours donc, mais qui n'ignore pas celui d'Althusser auquel est consacré, dans le même numéro, un article « critique mais admiratif » intitulé « La lecture de Marx » (J. Conilh).

Si donc on s'est mis à parler de "coupure " à propos de Saussure, c'est, tout en étant sensible à l'effervescence des théories poétiques, surtout par référence à une épistémologie plus laborieuse et moins expéditive, celle que mettaient en oeuvre Fichant et Pécheux dans Sur l'histoire des sciences (1969). C'est ainsi que, me référant au premier chapitre de cet ouvrage consacré à « la coupure galiléenne », je fus la première, du moins me semble-t-il, à poser, en 1970, ce genre de question au CLG :

«Sommes-nous avec Saussure devant une «coupure épistémologique» «constitutive d'une science», comparable à l'exemple généralement évoqué de Galilée? »(4)

Sans aller jusqu'à m'embourber dans un distinguo peu convaincant entre « rupture « et " coupure ", tel que le tentaient les auteurs précités, je ne m'octroyais pas encore, le droit de rêver sur cette métaphore, devenue toute naturelle et, pourtant, si curieuse. Pour dire le geste de démarcation cher aux positivistes, Althusser, qui d'ailleurs ne méprisait pas A. Comte, avait repris à Bachelard ce terme chargé d'affectivité et l'avait, si l'on peut dire, encore aiguisé : ce qui chez Bachelard était rupture avec les anciens objets et les confortables habitudes de la pensée était devenu coupure, tranchant dans le vif, dans le concret, par elle divisé entre l'observable, objet de science et la diversité d'un donné hétéroclite, laissé à la spéculation. Qu'il y ait là une violence n'échappait à personne et pas davantage aux lecteurs contemporains de Saussure; Schuchardt, dans son compterendu, a bien exprimé, en son temps, la révolte de l'empirisme malmené :

Avant d'en venir aux griefs particuliers que suscite en moi le système de Saussure, il me fallait mettre en avant le grief général que je nourris contre les systèmes qui procèdent par division et font violence aux choses. Bref Saussure lance un filet aux mailles serrées dans le torrent impétueux; en présence de ce dernier, je ne puis apercevoir clairement le filet ; ce qui unit fait toujours sur moi une impression plus forte, que ce qui sépare » (tr. P.Caussat,1978,175).

N'en était-il pas de même pour ceux qui refusaient de diviser Marx ? Toujours est-il que je m'autorisais ainsi non pas à diviser Saussure, mais à le séparer de ses contemporains, lui faisant mérite de l'isolement intellectuel dont il se plaignait.

On a beaucoup parlé du dogmatisme "théoriciste " souvent qualifié de "terroriste", mais on doit aussi rappeler les passions, aujourd'hui bien oubliées, dont ces discours étaient porteurs. Sous cette schématisation dont il est facile de se gausser, ce qui était ainsi ouvert, tranché, c'était une voie de sortie du ressassement universitaire et, pour beaucoup d'entre nous, c'était l'espoir global de changer le monde. Telle était la force rêvée de la théorie; elle ne se divisait pas; en tout domaine il importait qu'une analyse juste permît d'agir sur la réalité. C'est pourquoi il était aussi grave de se méprendre sur Saussure ou sur Freud que sur Marx. Nous étions mortellement sérieux.

31 Je ne me hasarderai pas à analyser les raisons de cette emprise massive du politique ; les textes montreraient, je crois, que même ceux qui se tenaient à l'écart de toute activité militante, acceptaient de débattre dans les termes qui en étaient inspirés, par exemple celui de "coupure» ou de "révolution». On peut comprendre alors l'ampleur des déceptions et des ressentiments et que l'évocation en reste étrangement passionnée. C'est 
avec irritation que Pavel dénonce « ce que la puissance de telles voix abrite d'immotivé, de fragile et de surfait » (24). Peut-être ! Mais il se trompe, selon moi, quand il affirme :

«A la fin des années 60 , la théorie à saveur linguistique fut mise au service des idéologies politiques du moment » (12).

Contrairement au schéma le plus fréquent la théorie n'était pas alors la servante de la politique ; elle avait sa propre force d'impulsion nourrie des retours conjugués à Marx et Freud, comme Althusser l'analysait dès 1964, dans la Nouvelle critique. Ces tentatives, nées dans l'analyse théorique, visaient à ébranler le dogmatisme des politiques; elles suffisaient en tout cas à les inquiéter. J’ajoute que la « désaffection » qui, dans les années 70, a consacré la fin du structuralisme, n'est pas sans rapport, me semble-t-il, avec la situation, cette fois entièrement politique, créée par la rupture entre les partis de gauche. «Coupure » disparait de la terminologie en même temps que l'espoir de la révolution.

Que tout cela ait été vécu dans une forme d'aveuglement n'a rien que de très ordinaire et de conforme au fonctionnement de l'idéologie. Il reste que tout ne s'est pas évanoui en fumée, puisqu'il y eut du travail qui a produit des effets en suscitant à son tour de nouveaux travaux; ce qui a tourné autour de la coupure saussurienne en fait partie. Mon propos n'était pas de reprendre ici ce que j'ai cherché à montrer ailleurs sur la pertinence de cette expression appliquée au texte de 1916, et encore moins m'y hasarderai-je à propos de l'édition critique. J'espère avoir montré simplement qu'elle a joué un rôle en donnant à certains l'envie de relire et de reprendre ce qui était jugé généralement réglé, leur permettant, alors qu'ils étaient souvent très loin des recherches spécialisées dans le domaine saussurien, de s'interroger sur ce texte et sur les évidences réductrices qu'on répétait sous son autorité. Oserai-je dire que la pensée de la "coupure » est encore susceptible de jouer ce rôle, à côté des travaux savants?

\section{NOTES}

1. Cf. J.-J. Courtine 1991.

2. Cf. entre autres M. Arrivé 1986 et F. Gadet 1987.

3. Cf. Meillet 1928.

4. Ce travail entrepris d'abord en cours a été poursuivi systématiquement dans le travail collectif du GRHIL (Groupe de recherche en histoire de la linguistique) commencé avec Langages n.49, 1978.

5. Cf. Normand 1982 "Les débuts de la linguistique structurale en France », LINX n.6.

6. Pour sa part le travail de F. Dosse, très précieux pour l'abondance de ses informations, me paraît faire trop de place aux souvenirs des témoins, c'est à dire à des reconstructions plus ou moins complaisantes.

7. Le CERM a éclaté comme tel, en même temps que disparaissait La Nouvelle Critique et qu'était rompue l'union de la Gauche, en 79-80). 